\title{
Consorcios microbianos con actividad ácido-láctica promisoria aislados desde inoculantes bacterianos nativos para ensilajes
}

\section{Microbial Consortiums with Promissory Lactic Acid Activity, Isolated from Native Bacterial Inoculants for Silages}

Fecha recepción: 06 de junio de 2013

Fecha Aprobación: 18 de octubre de 2013

\section{RESUMEN}

El objetivo de este trabajo fue aislar y caracterizar cepas de bacterias ácido lácticas (BAL) a partir de preparados microbianos (PM) nativos elaborados a base de residuos agroindustriales, como suero de leche, estiércol bovino y contenido ruminal, y diseñados como inoculantes biológicos para procesos de ensilaje de residuales orgánicos poscosecha. Se evaluaron 5 tratamientos (preparados microbianos, $\mathrm{PM}+33 \%$ de residuo inoculante), con 3 réplicas: 1) Tratamiento control sin inoculante (PMSI), 2) Suero de leche (PMSL), 3) Estiércol bovino (PMEB), 4) Combinación de suero + estiércol (PMM) y 5) Contenido ruminal (PMCR); además, en todos se adicionó: melaza $(20 \%)$, urea $(1 \%)$, sal mineral $(1 \%)$ y agua $(45 \%)$. En recipientes plásticos se fermentaron tapados durante 96 horas, a $14{ }^{\circ} \mathrm{C}$; de allí se obtuvieron 3 muestras de $500 \mathrm{~mL}$ de cada PM, se sembraron en
Byron Leoncio Díaz-Monroy ${ }^{1}$, Arabel Elías Iglesias², Elaine Valiño-Cabrera ${ }^{3}$

\begin{abstract}
This work aim was to isolate and characterize strains of $L A B$, from native microbial preparations (PM), using agroindustrial wastes as whey, bovine manure and rumen contents, designed as biological inoculants for silage processes of post harvest organic waste. Five treatments (microbial preparations, $\mathrm{PM}+33 \%$ inoculant residue) with 3 replications were evaluated: 1 ) Treatment control without inoculum (PMSI), 2) Whey (PMSL), 3) Cattle manure (PMEB), 4) Combination of Serum + manure (PMM), 5) Ruminal contents (PMCR). Also were added in all: $20 \%$ molasses, $1 \%$ urea, mineral salt $1 \%$, and water $45 \%$. They were fermented in plastic containers covered for 96 hours, at $14{ }^{\circ} \mathrm{C}$. hence 3 samples of $500 \mathrm{~mL}$ from each PM were obtained and planted in agar MRS (Man, Rogosa and Sharpe), incubated at $37{ }^{\circ} \mathrm{C}$ for $72 \mathrm{~h}$ under anaerobic conditions (AnaeroGen, Oxoid). LAB
\end{abstract}

Ph.D. Escuela Superior Politécnica de Chimborazo (Riobamba-Ecuador). Correo electrónico: bdiaz@espoch.edu.ec Ph.D. Instituto de Ciencia Animal (San José de las Lajas-Cuba). Correo electrónico: aelias@ica.co.cu

Ph.D. Instituto de Ciencia Animal (San José de las Lajas-Cuba). Correo electrónico: evalino@ica.co.cu 
agar MRS (Man, Rogosa y Sharpe) y se incubaron a $37^{\circ} \mathrm{C}$ durante $72 \mathrm{~h}$ bajo condiciones anaerobias (AnaeroGen, Oxoid). Las cepas de BAL son catalasa y oxidasa negativas, cocos, coco-bacilos y bacilos Gram positivos no esporulados. La identificación bioquímica se realizó con el sistema API-50CHL (BioMérieux, France) y la base de datos APILAB Plus versión 3.3.3. Se aislaron 39 cepas de BAL, 4 del PMSI (2 Lactobacillus plantarum, 1 L. brevis y 1 Lactococcus lactis), 12 del PMSL (3 Lactobacillus rhamnosus, 3 L. casei, 2 L. acidophilus, 2 L. paracasei y 2 L. fermentum), 7 del PMEB (3 Streptococcus bovis, 2 Lactobacillus plantarum y 2 Leuconostoc mesenteroides), 10 del PMM (3 Lactobacillus casei, 2 L. plantarum, 2 L. acidophilus, 1 L. fermentum, 1 L. rhamnosus y 1 L. paracasei) y 6 del PMCR ( 2 Lactobacillus vitulinus, 2 L. ruminus, 1 L. reuteri y 1 Pediococcus damnosus). En conclusión, la mejor fuente de cepas de BAL fue PMSL, que le otorga ventajas como posible inoculante bacteriano para ensilajes. En todos los PM se identificaron cepas homofermentativas y heterofermentativas, asociadas en consorcios microbianos; se generó una alternativa tecnológica para aprovechar tres residuos agroindustriales.

Palabras clave: Bacterias ácido lácticas, BAL, Preparado microbiano, PM, Suero de leche, Estiércol bovino, Contenido ruminal. strains are catalase and oxidase negative, coco, coco-bacilli and gram-positive non-spore forming. The biochemical identification was performed with the API-50 CHL (BioMérieux, France) system and the database APILAB Plus version 3.3.3.

39 strains LAB were isolated, 4 from PMSI (2 Lactobacillus plantarum, 1 L. brevis, 1 Lactococcus lactis), 12 from PMSL (3 Lactobacillus rhamnosus, 3 L. casei, 2 L. acidophilus and 2 L. paracasei and 2 L. fermentum), 7 from PMEB (3 Streptococcus bovis, 2 Lactobacillus plantarum and 2 Leuconostoc mesenteroides), 10 from PMM (3 Lactobacillus casei, 2 L. plantarum, 2 L. acidophilus, 1 L. fermentum, 1 L. rhamnosus and 1 L. paracasei) and 6 from PMCR (2 Lactobacillus vitulinus, 2 L. ruminus, 1 L. reuteri and 1 Pediococcus damnosus). As conclusion the best source of LAB strains was PMSL, which has the advantage to be a possible bacterial silage inoculant. In all PM were identified homofermentative and heterofermentative $\mathrm{LAB}$ strains, associated in microbial consortia. Aditionnally a technological alternative was created to profit from three new agro-industrial wastes.

Key Words: Lactic acid bacteria,LAB, Microbial preparation, MP, Whey, Bovine Manure, Ruminal Content. 


\section{INTRODUCCIÓN}

Los lactobacilos son bacterias Gram positivas, anaeróbicas o aeróbicas facultativas, que aparecen en grandes cantidades en la mayor parte del tracto gastrointestinal; se ubican generalmente en lugares donde hay gran variedad de sustancias ricas en carbohidratos disponibles (1). En un estudio con suero delechecosteño, quecorrespondea unafermentación espontánea de leche en calabazos (fruto seco de Lagenaria vigalis), por acción de bacterias ácido lácticas $(\mathrm{BAL})$ y otros microorganismos, se aislaron siete cepas con características probióticas, según clasificación API ${ }^{\circledR} 50 \mathrm{CHL}$, que son: Enterococcus durans 381, E. faecium 02, Lactobacillus fermentum, L. fermentum 72, L. fermentum 1-1, L. fermentum 75 y L. rhamnosus 73 (2). Las cepas de BAL aisladas de la etapa temprana de la fermentación natural de aceitunas verdes no desamargadas fueron Lactobacillus plantarum, Lactobacillus pentosus, Pediococcus pentosaceus y Lactobacillus brevis; estas especies son de origen natural en la fermentación de este producto (3). A partir de la identificación bioquímica fue posible identificar en la leche de oveja guirra las especies Lactobacillus acidophilus, L. brevis, L. delbrueckii lactis, L. delbrueckii delbrueckii, L. paracasei paracasei, L. pentosus, L. plantarum, L. rhamnosus, Lactococcus lactis subsp lactis, Lactococcus raffinolactis, Leuconostoc mesenteroides mesenteroides y Pediococcus pentosaceus (4).

Como se puede observar, la búsqueda de microorganismos promisorios con actividad ácido-láctica ha generado resultados positivos en múltiples procesos de fermentación, al ser utilizados como inóculos. La Escuela Superior Politécnica de Chimborazo del Ecuador tiene interés por desarrollar tecnología propia en el ámbito de los aditivos biológicos para el proceso de ensilaje, por ello es necesario aislar, caracterizar y evaluar cepas bacterianas autóctonas que tengan información genética para trabajar y adaptarse a sustratos también autóctonos y abundantes en nuestro medio, lo cual resulta de gran interés científico, tecnológico, económico y social; estas cepas forman parte de diversos nichos ecológicos que la naturaleza ha diseñado, y están disponibles para ser estudiados y aprovechados.
El objetivo de este trabajo fue aislar y caracterizar cepas de BAL, a partir de preparados microbianos (PM) nativos elaborados a base de residuos agroindustriales, como suero de leche, estiércol bovino y contenido ruminal, y diseñados como inoculantes biológicos para procesos de ensilaje de residuales orgánicos poscosecha.

\section{MATERIALES Y MÉTODOS}

Elaboración de los preparados microbianos (PM). Los ingredientes (Tabla I) se mezclaron y homogeneizaron en recipientes plásticos con capacidad para $30 \mathrm{~L}$, y se mantuvieron tapados herméticamente durante 96 horas, a temperatura ambiente del sitio de investigación $\left(14{ }^{\circ} \mathrm{C}\right.$, en Riobamba, Ecuador); durante este tiempo se evaluó la dinámica de la fermentación y se determinaron cronológicamente los indicadores referentes a ella.

Tabla I. Dosificación de ingredientes para el preparado microbiano

\begin{tabular}{|c|c|}
\hline Materias primas & $\mathbf{\%}$ \\
\hline SL o EB o CR & 33 \\
\hline Melaza de caña & 20 \\
\hline Urea & 1 \\
\hline Sal mineral (bovinos) & 1 \\
\hline Agua & 45 \\
\hline Total & 100 \\
\hline
\end{tabular}

SL: Suero de leche de vaca; EB: Estiércol bovino fresco; CR: Contenido ruminal de bovino

Todos los tratamientos tuvieron la misma formulación, únicamente cambiaron el tipo de inoculante.

Recolección y procesamiento de las muestras. En frascos estériles de vidrio ámbar, con taparrosca, se tomaron tres muestras de $500 \mathrm{~mL}$ de cada uno de los 3 preparados microbianos homogeneizados, es decir, en total se recolectaron 9 muestras. En el laboratorio, estos frascos se colocaron en un agitador orbital durante 5 minutos a $100 \mathrm{rpm}$, para lograr la homogeneización y distribución uniforme de los microorganismos en el medio.

Aislamiento y caracterización de las cepas. Con las muestras de los preparados microbianos se prepararon diluciones $10^{-1}$ en agua de peptona ( $1 \%$ ) 
(Sigma); luego, se hicieron diluciones sucesivas en agua con el aditivo Tween $80(4 \%)$ hasta $10^{-6}$, y se homogeneizaron en agitador orbital, durante 5 minutos a 100 rpm; después, se agitaron en auto vortex durante $1 \mathrm{~min}$. y se sembraron en agar MRS (Man, Rogosa y Sharpe). Las placas fueron incubadas a $37{ }^{\circ} \mathrm{C}$ durante $72 \mathrm{~h}$ bajo condiciones anaerobias (AnaeroGen, Oxoid).

Las cepas ácido-lácticas fueron identificadas fenotípicamente mediante tinción, según Gram, pruebas de la catalasa y oxidasa, apariencia de colonia y morfología celular. Se seleccionaron las colonias catalasa y oxidasas negativas y con morfología de cocos, coco-bacilos y bacilos Grampositivos no esporulados.

El estudio del metabolismo de los carbohidratos se realizó con el sistema comercial API-50 CHL (BioMérieux, France), según las instrucciones del fabricante (Figura 1); cada cepa seleccionada proveniente de agar MRS se inoculó en medio
$50 \mathrm{CH}$ a una concentración de McFarland 2. Se Ilenaron los pocillos de la galería; sus cúpulas se cubrieron con vaselina, para crear condiciones de anaerobiosis, y se incubaron a $37^{\circ} \mathrm{C}$ durante 48 horas. El cambio de color del pocillo indica la formación de ácidos que disminuyen el $\mathrm{pH}$. Los perfiles obtenidos en la fermentación de carbohidratos se registraron en la base de datos APILAB Plus versión 3.3.3 (bioMérieux, Marcy I'Etoile, Francia), y se obtuvo la identificación a nivel de género y especie de cada cepa.

Lactobacillus casei ATCC 393, Lactobacillus salivarius ATCC 11741, Enterococcus faecium ATCC 19434 y Pediococcus damnosus ATCC 25249 se utilizaron como cepas de referencia.

Los cultivos puros de bacterias ácido-lácticas se conservaron en tubos con agar MRS en refrigeración $\left(5 \pm 2{ }^{\circ} \mathrm{C}\right)$ y en caldo MRS, con la adición del $15 \%$ (v/v) de glicerol en congelación a $-80{ }^{\circ} \mathrm{C}$.

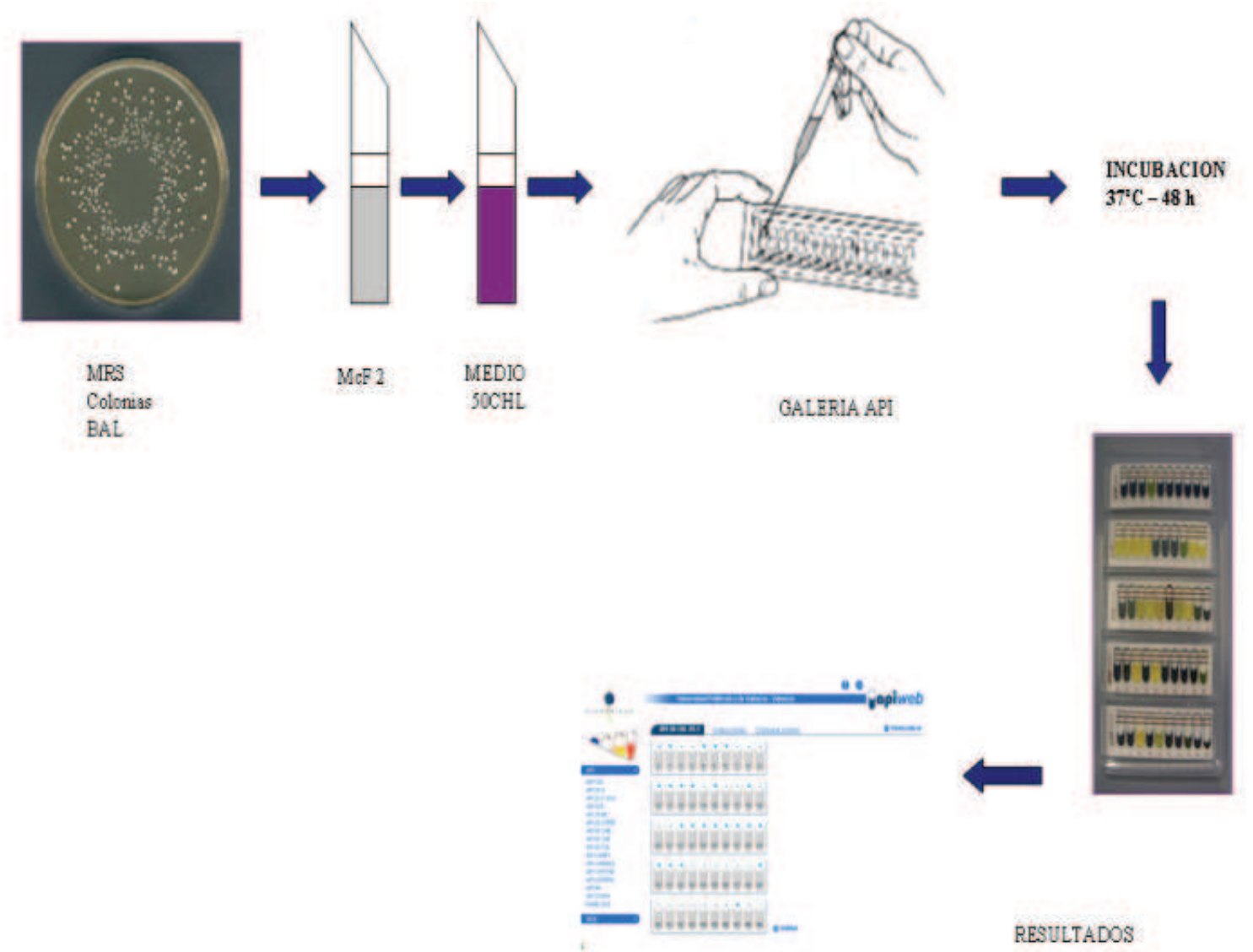

Figura 1. Esquema de trabajo con el sistema API $50 \mathrm{CHL}$ 


\section{RESULTADOS Y DISCUSIÓN}

Se aislaron en total 39 cepas de BAL (Tabla II): 4 del PMSI (10,26\%), 12 del PMSL $(30,77 \%), 7$ del PMEB (17,95\%), 10 del PMM (25,64\%) y 6 del PMCR $(15,38 \%)$.

Beldarraín et al. (5) indicaron que actualmente se dispone de muchos medios de cultivo para el aislamiento y diferenciación de las bacterias lácticas, aunque solo a algunos de ellos se les considera selectivos, entre ellos está MRS, para Lactobocillus spp. Este medio de cultivo permitió el crecimiento de todas las especies de BAL en este estudio. Las bacterias en un cultivo expresan sus características visibles y medibles en el laboratorio; esta metodología, a pesar de que resulta en ocasiones insuficiente para la correcta identificación de las especies, sigue vigente y ha sido ampliamente utilizada en la identificación bacteriana (6). La insuficiencia de la metodología de caracterización basada en el fenotipo se debe principalmente a la inestabilidad de las características fenotípicas de las BAL, lo cual puede estar relacionado con la presencia de plásmidos, fenómeno que obstaculiza estas pruebas en su taxonomía (7).

La caracterización bioquímica se basa en las respuestas del microorganismo a la fermentación de azúcares, y permite diferenciar entre especies de Lactobacillus (8). En las BAL, la ruta más estudiada es la de la lactosa, y la capacidad de fermentación varía entre las diferentes especies lácticas (9).

Los sistemas comerciales que pueden ser usados para la caracterización bacteriana son API 20 STREP, API 50 CHL, VITEK 2 COMPACT (bioMérieux, France), Diabts (Rosco, Denmark) y BIOLOG GP Micro Plate (BIOLOG Inc US), Ios cuales ofrecen bases de datos para la identificación de microorganismos determinados (7).

Específicamente en el caso de las $\mathrm{BAL}$, muchos autores han empleado la técnica API 50 Biomeriux para la identificación bioquímica (10). Por lo que es habitual la identificación de BAL aisladas de diferentes matrices mediante este sistema comercial (11 - 13).

Pese a ello, la identificación a nivel de cepa es importante cuando se caracterizan probióticos, porque los efectos beneficiosos que puedan tener estas bacterias sobre la salud no se pueden atribuir de forma generalizada a un género o especie, sino que dependen de la cepa (4).

La caracterización bacteriana desarrollada en este estudio permitió aislar cuatro cepas BAL del PMSI: dos de Lactobacillus plantarum (50\%), una de Lactobacillus brevis y otra de Lactococcus lactis ( $25 \%$ cada una del total del grupo). Estas cepas juntas en un mismo nicho ecológico conforman un consorcio microbiano, una asociación natural de dos o más poblaciones microbianas de diferentes especies, que actúan conjuntamente como una comunidad en un sistema complejo, donde todos se benefician de las actividades de los demás. La asociación refleja estilos de vida sinérgicos o sintróficos (que significa "comiendo juntos"), en los que el crecimiento y el flujo cíclico de nutrientes se conducen más efectiva y eficientemente que en poblaciones individuales (14).

En el PMSL se identificaron 12 cepas de BAL, con predominio de Lactobacillus rhamnosus y Lactobacillus casei (tres cepas de cada especie, es decir, 25\% de cada una), seguidas de Lactobacillus acidophilus, Lactobacillus paracasei y Lactobacillus fermentum (dos cepas de cada especie, es decir, 16,67\% cada una) (Figura 2). En este PMSL existe mayor actividad bacteriana, Io que ratifica lo expuesto por (15), quienes sostienen que los cultivos bacterianos mixtos pueden tener funciones complicadas que poblaciones individuales no podrían; además, la vida en asociación puede generar mayor resistencia a las fluctuaciones del ambiente y promover la estabilidad de los miembros en el tiempo. 


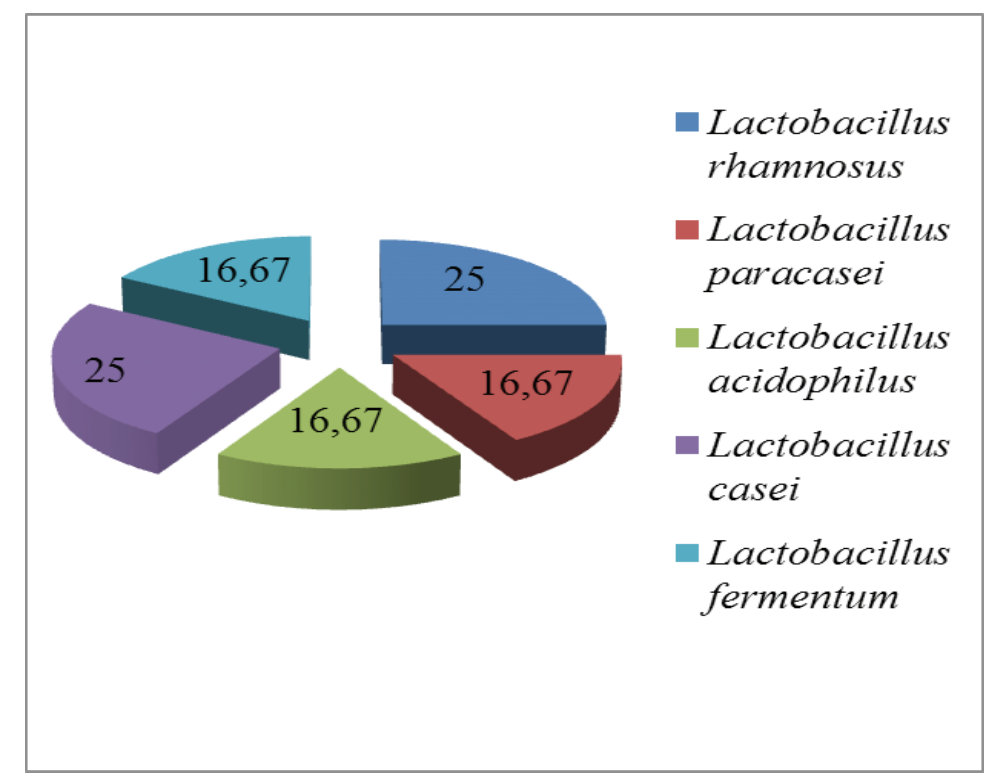

Figura 2. Distribución de cepas de bacterias ácido-lácticas en el preparado microbiano de suero fresco de leche

Estos rasgos distintivos dependen de dos características: primera, los miembros de un consorcio se comunican ya sea por el intercambio de sustancias o por señales moleculares; cada población detecta y responde a la presencia de otras dentro del consorcio, al ejercer sobre ellas un control positivo o negativo en su crecimiento o metabolismo o en ambos. Esta comunicación permite la segunda característica importante: la división del trabajo; así, la producción total de un consorcio depende de la combinación de tareas desempeñadas por los constituyentes individuales, es decir, por las poblaciones microbianas involucradas. Otra importante característica de los consorcios es su habilidad para desempeñar funciones que requieren múltiples pasos.

En el PMEB se identificaron 7 cepas de BAL: 3 de Streptococcus bovis (42,86\%), 2 de Lactobacillus plantarum y 2 de Leuconostoc mesenteroides (28,57\% de cada especie). Se observa nuevamente la presencia de un consorcio microbiano.

En el PMM se identificaron 10 cepas de BAL: 3 de Lactobacillus casei (30\%), 2 de Lactobacillus plantarum y 2 de Lactobacillus acidophilus (20\% de cada una), una de Lactobacillus fermentum, 1 de Lactobacillus rhamnosus y 1 de Lactobacillus paracasei (10\% de cada una).
En el PMCR se aislaron 6 cepas de BAL: dos de Lactobacillus vitulinus y dos de Lactobacillus ruminus, (33,33\% de cada cepa), 1 de Lactobacillus reuteri y 1 de Pediococcus damnosus (16,67\% de cada una). Tal como se observa en la Tabla II, las especies de BAL aisladas corresponden en su mayoría al género Lactobacillus sp., con 34 cepas $(87,18 \%)$, lo cual determina la importancia de este grupo en procesos de fermentación ácidoláctica; le sigue el género Enterococcus sp., con 3 cepas (7,70\% de total); luego aparecen los géneros Lactococcus sp. y Pediococcus sp., con una cepa en cada caso $(2,56 \%$ para cada género).

Por otro lado, se ha verificado la presencia de diferentes cepas de BAL, que se agrupan en homofermentativas o heterofermentativas, con base en el producto final de su fermentación (15).

Tabla II. Distribución por género de bacterias ácido-lácticas aisladas desde los preparados microbianos

\begin{tabular}{|c|c|c|}
\hline Género bacteriano & $\mathbf{N}^{\circ}{ }^{\circ}$ de cepas & \% del total \\
\hline Lactobacillus sp. & 34 & 87,18 \\
\hline Lactococcus sp. & 1 & 2,56 \\
\hline Enterococcus sp. & 3 & 7,70 \\
\hline Pediococcus sp. & 1 & 2,56 \\
\hline Total & 39 & 100 \\
\hline
\end{tabular}


Las homofermentativas, como Lactococcus, Streptococcus, Pedicococcus, Vagococcus y algunos Lactobacillus, poseen la enzima aldolasa y producen ácido láctico como producto principal de la fermentación de la glucosa, utilizando la vía de glucólisis (Embden-Meyerhof) (16). Por su parte, los géneros Leuconostoc, Oenococcus, Weisella, Carnobacterium, Lactosphaera y algunos Lactobacillus son heterofermentativos y convierten hexosas a pentosas por la vía 6-fosfogluconatofosfocetolasa, por lo que además de ácido láctico generan otros productos como acetato, etanol y CO2 (17). En cambio, las heterofermentativas facultativas tienen la capacidad de utilizar ambas vías, siendo homofermentativo su metabolismo principal; si se modifican algunas condiciones de cultivo, tales como la concentración de glucosa, $\mathrm{pH}$ y la restricción de nutrientes, se induce la vía 6-PG/PK, causando la fermentación heteroláctica (18).

En esta investigación se identificaron cepas homofermentativas, heterofermentativas obligadas y heterofermentativas facultativas; su distribución se observa en la Tabla III.

Tabla III. Distribución de cepas de bacterias ácido-lácticas aisladas de preparados microbianos, según su afinidad metabólica de fermentación

\begin{tabular}{|c|c|c|}
\hline Tipo de metabolismo/Cepas & N. ${ }^{\circ}$ de cepas & \% del total \\
\hline $\begin{array}{c}\text { Homofermentativa: Lactococcus lactis (1), } \\
\text { Lactobacillus rhamnosus (4), Lactobacillus } \\
\text { paracasei (3), Lactobacillus acidophilus (4), } \\
\begin{array}{c}\text { Enterococcus faecium (3), Lactobacillus salivarius } \\
\text { (2), Lactobacillus vitulinus (2), Lactobacillus } \\
\text { ruminus (2), Pediococcus damnosus (1) }\end{array}\end{array}$ & 22 & 56,41 \\
\hline $\begin{array}{c}\text { Heterofermentativa obligada: Lactobacillus brevis } \\
\text { (1), Lactobacillus fermentum (3), Lactobacillus } \\
\text { reuteri (1) }\end{array}$ & 5 & 12,82 \\
\hline $\begin{array}{c}\text { Heterofermentativa facultativa: Lactobacillus } \\
\text { plantarum (6), Lactobacillus casei (6) }\end{array}$ & 12 & 30,77 \\
\hline Total & 39 & 100 \\
\hline
\end{tabular}

La diferencia de una vía a otra viene marcada por la presencia o ausencia de la enzima aldolasa, que es clave en la glucólisis; las heterofermentativas carecen de aldolasa y no pueden romper la fructosa 1,6-difosfato, en su lugar, oxidan la glucosa 6-fosfato hasta 6-fosfogluconato y después lo descarboxilan hasta xilulosa 5-fosfato, que se escinde hasta gliceraldehído 3-fosfato y acetil-fosfato por medio de la fosfocetolasa; el gliceraldehído 3-fosfato se convierte en ácido láctico con la producción de una molécula de ATP (adenosina trifosfato), mientras que el acetil-fosfato acepta electrones del $\mathrm{NADH}$ (nicotinamida adenina dinucleótido) que se ha generado durante la formación de xilulosa 5-fosfato, dando lugar directamente a etanol sin producir ATP. Por ello, las heterofermentativas producen solamente $1 \mathrm{~mol}$ de ATP de la glucosa, en lugar de 2, como hacen las homofermentativas. Como las heterofermentativas descarboxilan el 6-fosfogluconato, producen $\mathrm{CO}_{2}$ como producto de fermentación (20).

Las BAL tienen varios mecanismos antagónicos contra patógenos, entre ellos se destaca la producción de bacteriocinas, que son derivados del metabolismo, con función antimicrobiana, de naturaleza peptídica, sintetizadas ribosomalmente y que afectan a bacterias relacionadas con las que las producen (1). La obtención de BAL útiles para ensilajes debe considerar que las mejores cepas provienen de sustratos semejantes y que la cepa debe probarse en condiciones ecológicas semejantes a las anteriores a ser aislada (21). 


\section{CONCLUSIONES}

La formulación de ingredientes para los preparados microbianos fue apropiada, se logró producir altas concentraciones de cepas nativas de BAL.

La mejor fuente de cepas de BAL fue el preparado microbiano a base de suero fresco de leche, lo cual le otorga características adecuadas como futuro inoculante bacteriano para ensilajes.

En todos los preparados microbianos se evidenció la presencia de consorcios microbianos y no de especies únicas de BAL, lo cual presenta ventajas para el trabajo posterior de inoculación en ensilajes. Se generó una alternativa tecnológica para el aprovechamiento de tres residuos agroindustriales, lo cual es un aporte a la mitigación de la contaminación ambiental.

\section{AGRADECIMIENTOS}

A la Secretaría Nacional de Educación Superior, Ciencia, Tecnología e Innovación, "SENESCYT", del Ecuador, por el financiamiento de esta investigación a través de una beca para estudios de Doctorado; a la Escuela Superior Politécnica de Chimborazo, "ESPOCH", por el auspicio de este trabajo, y al Instituto de Ciencia Animal, "ICA", de Cuba, por su asesoramiento y tutoría permanentes.

\section{REFERENCIAS}

(1) Jaramillo D, Meléndez A, Sánchez O. Evaluación de la producción de bacteriocinas a partir de Lactobacilos y Bifidobacterias. Rev Ven Cien y Tec Alimentos 2010; 1 (2): 193-209.

(2) Vigil M, Acuña Y, Valenzuela J. Evaluación in vitro del potencial probiótico de bacterias ácido lácticas aisladas de suero costeño. Actual Biol. 2010; 32(93):129-138.

(3) Rodríguez H, Curiel J, Landete J, De las Rivas B, De Felipe F, Cordoves C, Mancheno J y Muñoz R. Food Phenolics and Lactic Acid Bacteria. Int. J. Food Microbiol. 2009; 132: 79-90.

(4) Amorocho C. Caracterización y potencial probiótico de bacterias lácticas aisladas de leche de oveja Guirra. Tesis doctoral. Universitat Politècnica de València. España. 2011.

(5) Beldarraín T, González A y Kala D. Métodos de identificación de cepas productoras de ácido láctico aisladas a partir de matrices alimentarias. Cien y Tec de Alimentos 2011; 21(1): 72-77.

(6) Ruiz P. Biodiversidad de la microbiota láctica presente en la fermentación maloláctica de vinos tintos de la variedad cencibel: caracterización molecular y tecnológica para la selección de cepas. Tesis doctoral. Universidad de Castilla-La Mancha. Toledo, España. 2010.

(7) Pot B and Tsakalidou E. Taxonomy and Metabolism of Lactobacillus. En Lactobacillus Molecular Biology. From genomic to probiotic. 2009.

(8) McLeod A, LudvigNyquist O, Snipen L, Naterstad K., Axelsson L. Diversity of lactobacillus sakei strains investigated by phenotypic and genotypic methods. Systematic and Applied Microbiology 2008; (31): 393-403.

(9) Hernández A. Lactococina 972: Caracterización genética, modo de acción y optimización de la producción en biorreactores. Tesis Doctoral. Universidad de Oviedo. España. 2005.

(10) Rodríguez M, Chacon Z, Guerrero B, Rojas J y López G. FCV-LUZ XVII 2007; (6): 641-646.

(11) Ouoba L, Nyanga-Koumou C, Parkouda C, Sawadogo H, Kobawila S, Keleke S, Diawara B, Louembe D, Sutherland J. Genotypic Diversity of Lactic Acid Bacteria Isolated from African Traditional Alkaline-Fermented Foods. Journal of Applied Microbiology 2009; (108): 2019-2029.

(12) Todorov S and Dicks L. Bacteriocin Production by Pediococcus pentosaceus Isolated from Marula (Scerocarya birrea). International Journal of Food Microbiology 2009; (132): 117-126.

(13) Ávila J, Ávila M, Tovar B, Brizuela M, Perazzo Y, Hernández H. Capacidad probiótica de cepas del género Lactobacillus extraídas del tracto intestinal de animales de granja. Rev Cien y Tec alimentos 2010; (2): 161-169.

(14) Ingraham J e Ingraham C. Introducción a la Microbiología. 2007; Volumen 2. Edit. Reverté. Barcelona, España. 
(15) Madigan MT, Martinko JM, Parker J. Biología de los microorganismos. 2004. $10^{\mathrm{a}}$ ed. Ed. Prentice Hall. Madrid, España.

(16) Axelsson L. Lactic Acid Bacteria: Classification and Physiology. En: Lactic Acid Bacteria, Microbiology and Functional Aspects. 1998. $2^{\text {nd }}$ edition. Pp. 1-72. Marcel Dekker Inc. New York, USA.

(17) Ramírez C, Ulloa P, Velázquez M, Ulloa J, Romero F. Bacterias lácticas: importancia en alimentos y sus efectos en la salud. Revista Fuente 2011; 2(7).

(18) Axelsson L. Lactic Acid Bacteria: Classification and Physiology. En: Lactic Acid Bacteria: Microbiological and Functional Aspects. 2004. 3rd rev. and exp. Ed. Marcel Dekker, Inc., New York.
(19) Prescott LM, Harley JP, Klein DA. Microbiología. 4. ${ }^{\text {a }}$ ed., McGraw-Hill Interamericana. 1999. Zaragoza, España.

(20) Mora N y García A. Susceptibilidad de bacterias ácido lácticas (BAL) frente a diversos antibióticos. Tesis licenciado química de alimentos. Universidad Autónoma del Estado de Hidalgo. México. 2007.

(21) Rosmini M, Sequeira G, Guerrero-Legarreta I, Martí L, Dalla-Santina R, Frizzo L y Bonazza J. Producción de probióticos para animales de abasto: Importancia del uso de la microbiota intestinal indígena. Rev Mex Ing Quím. 2004; (3): 181-191. 